\title{
Kanouté, F. et Charette, J. (dir.) (2018). La diversité ethnoculturelle dans le contexte scolaire québécois: Pratiquer le vivre-ensemble. Montréal, QC : Presses de I'Université de Montréal.
}

\section{RECENSION}

Sous la direction de Fasal Kanouté et de Josée Charette, cet ouvrage vise à mettre en valeur différentes contributions issues du milieu de la recherche concernant les enjeux de la diversité en contexte scolaire. Pour ce faire, près d'une vingtaine d'auteurs, provenant du milieu de l'éducation ou de la recherche en service social, se sont unis au sein de ce projet de livre afin de mettre en avant les résultats de leurs projets d'études respectifs.

\section{Présentation}

Louvrage dirigé par Kanouté et Charette (2018) propose onze chapitres distincts abordant la thématique de la diversité ethnoculturelle en contexte scolaire québécois. Ces chapitres se répartissent en trois sections distinctes. Au sein de la première section, quatre chapitres contribuent à documenter le cheminement scolaire de l'élève immigrant. Pour ce faire, le rapport à l'école des jeunes immigrants tout comme les défis et les éléments facilitateurs de leur intégration au sein du système scolaire québécois sont mis de l'avant. De plus, le rôle de l'activité artistique, selon une approche collaborative, dans la structuration et la prise en charge de leur identité est traité en profondeur. Finalement, un quatrième chapitre conclut cette première partie de l'ouvrage en décrivant les défis et les enjeux associés à un retour aux études des élèves immigrants. 
Pour sa part, la deuxième partie du livre aborde la thématique de la prise en compte de la diversité en milieu scolaire. En abordant les résultats de leur projet de recherche, les auteurs ayant contribué à la rédaction de cette partie de l'ouvrage abordent les différentes approches pédagogiques (éducation inter/multiculturelle, virage antiraciste, éducation à la citoyenneté) qui se sont succédé dans l'histoire du Québec quant à la considération de la diversité sociale, ethnoculturelle et linguistique des élèves. Concernant l'apport respectif des différents chapitres à l'ouvrage, le premier texte aborde spécifiquement le thème de l'éducation interculturelle et inclusive par rapport à l'enseignement de la culture religieuse à l'école. Le deuxième chapitre traite quant à lui des modalités de leaderships mises en ouvre par des directeurs d'établissements scolaires montréalais afin de faciliter l'inclusion des élèves immigrants au sein du réseau scolaire. Finalement, le dernier texte de cette partie dresse un portrait ethnographique des écoles caractérisées comme étant «inclusives».

Enfin, la troisième partie de l'ouvrage aborde spécifiquement les défis reliés à la prise en compte de la diversité. Pour ce faire, les points de vue de différents intervenants sont mis en lumière par les résultats de recherche issus des projets des différents auteurs ayant contribué à la rédaction de cette partie. Dans un premier temps, le thème de l'implication de parents maghrébins dans le cheminement scolaire d'élèves immigrants est détaillé. Ensuite, une deuxième étude présente le rôle des intervenants communautaires scolaires interculturels (ICSI) quant à l'intégration des parents et des élèves immigrants. Cette étude est suivie par un troisième texte traitant du parcours biographique d'intervenants communautaires travaillant en milieu pluriethnique. Cette section du livre est complétée par un texte traitant des principaux défis découlant de l'insertion socioprofessionnelle d'enseignants immigrants ayant reçu leur formation initiale en éducation à l'étranger.

\section{Forces et faiblesses de l'ouvrage}

À mon sens, le collectif dirigé par Kanouté et Charette (2018) saura combler les attentes du lecteur s'intéressant à la thématique de la diversité ethnoculturelle en contexte scolaire québécois. En effet, le partage des différents champs d'expertise des auteurs qui ont été interpelés afin de contribuer à la rédaction de cet ouvrage permet de répondre aux attentes d'un lectorat élargi. Personnellement, j'ai apprécié l'arrimage entre les auteurs issus du milieu des sciences de l'éducation ainsi que les collaborateurs provenant du domaine des sciences sociales, et ce, afin de présenter une importante diversité de chapitres.

De plus, la variété des sujets abordés au sein de l'ouvrage permet au lecteur, qui n'est pas un spécialiste dans le domaine, de s'approprier les fondements théoriques liés à la thématique de la diversité ethnoculturelle ainsi que d'accéder facilement à des résultats vulgarisés issus d'études empiriques réalisées en milieu scolaire. À cet effet, je conçois que le collectif piloté par Kanouté et Charette (2018) constitue une contribution importante au milieu de l'éducation, et ce, en rassemblant divers textes scientifiques qui pourront éventuellement œuvrer en tant que compléments d'information pour le Cadre de référence sur l'accueil et l'intégration des élèves issus de l'immigration au Québec (2014) récemment publié par le ministère de l'Éducation. 
Par ailleurs, je crois que les retombées de l'ouvrage auraient pu être un peu plus grandes si les différents auteurs avaient donné davantage de précisions concernant la rigueur des devis méthodologiques mis de l'avant à l'intérieur de chacune des recherches présentées. En effet, la structure du collectif fait en sorte qu'il est difficile pour le lecteur d'être critique à l'égard des résultats scientifiques présentés puisque très peu d'informations permettent de décrire les modalités sous-jacentes à l'analyse des données qui a été réalisée. De plus, la très grande majorité des études présentées ont mis de l'avant un protocole d'étude se rapportant à la recherche qualitative. Ce constat m'a amené à me demander si la mise en place d'études quantitatives aurait pu permettre de détailler davantage la thématique de la diversité ethnoculturelle en mettant en lumière des résultats d'une autre nature qui auraient peut-être permis de répondre à une classe différente d'objectifs et de questions de recherche.

\section{Pour citer cet article}

Rajotte, T. (2019). Kanouté, F. et Charette, J. (dir.) (2018). La diversité ethnoculturelle dans le contexte scolaire québécois : Pratiquer le vivre-ensemble. Montréal, QC : Presses de l'Université de Montréal. Formation et profession, 27(1), 131-134. http://dx.doi.org/10.18162/fp.2019.168 